ALEA, Lat. Am. J. Probab. Math. Stat. 15, 549-569 (2018)

DOI: $10.30757 /$ ALEA.v15-22

\title{
Swap-invariant sequences and random measures
}

\author{
Felix Nagel \\ University of Berne, \\ Institute of Mathematical Statistics and Actuarial Science, \\ Alpeneggstrasse 22, \\ 3012 Berne, Switzerland. \\ E-mail address: felix.nagel@stat.unibe.ch
}

\begin{abstract}
In this work we analyze the concept of swap-invariance, which is a weaker variant of exchangeability. An integrable random vector $\xi$ in $\mathbb{R}^{n}$ is called swap-invariant if $\mathbf{E}\left|\sum_{j} u_{j} \xi_{j}\right|$ is invariant under all permutations of $\left(\xi_{1}, \ldots, \xi_{n}\right)$ for each $u \in \mathbb{R}^{n}$. A random sequence is called swap-invariant if all finite dimensional subsequences are swap-invariant. It has been shown by Molchanov et al. (2014) that the ergodic theorem holds for swap-invariant sequences. We derive two representations of swap-invariant sequences in terms of certain ergodic limits and of a sequence that is exchangeable under an appropriate probability measure. Swapinvariance can be extended to random measures on $[0,1]$ or $\mathbb{R}_{+}$. Using the results for sequences, it is shown that the ergodic theorem holds for swap-invariant random measures. This allows us to derive a representation in terms of the ergodic limit and an exchangeable random measure.
\end{abstract}

\section{Introduction}

Two integrable random vectors $\xi$ and $\eta$ in $\mathbb{R}^{n}$ are called zonoid equivalent if $\mathbf{E}|\langle u, \xi\rangle|=\mathbf{E}|\langle u, \eta\rangle|$ for every $u \in \mathbb{R}^{n}$, where $\langle\cdot, \cdot\rangle$ denotes the Euclidean inner product. A vector $\xi$ of integrable random variables is called swap-invariant if $\xi \circ \pi$ and $\xi$ are zonoid equivalent for all permutations $\pi$ of $\{1, \ldots, n\}$. Swap-invariance is weaker than exchangeability. Both exchangeability and swap-invariance are extended to random sequences by requiring the respective properties for all finitedimensional distributions. Swap-invariant sequences were introduced by Molchanov et al. (2014). They prove that a swap-invariant sequence $\xi$ satisfies the ergodic theorem, that is $n^{-1} \sum_{j=1}^{n} \xi_{j} \rightarrow X$ almost surely as $n \rightarrow \infty$ for some random variable $X$, see their Theorem 17. For exchangeable sequences this is a consequence of

Received by the editors May 17th, 2016; accepted March 22th, 2018.

2010 Mathematics Subject Classification. 28D99, 37A50, 60F25, 60G09, 60G57.

Key words and phrases. Ergodic theorem, exchangeability, random measure, swap-invariance, zonoid equivalence.

Research supported by Swiss National Science Foundation Grant 200021-153597. 
de Finetti's theorem. In Molchanov et al. (2014) it is shown that a swap-invariant sequence can be related to an exchangeable sequence by a transformation and a simultaneous change of the probability measure. Their proof is based on a result by Hardin (1981), Theorem 1.1, that the distribution of an integrable random vector $\xi$ in $\mathbb{R}^{n}$ is uniquely determined by the absolute moments $\mathbf{E}|1+\langle u, \xi\rangle|$ for all $u \in \mathbb{R}^{n}$.

Section 2 is devoted to a detailed analysis of the connections between swapinvariant and exchangeable sequences. First it is shown that each swap-invariant sequence of random variables with only two positive values is exchangeable. Further we present a simple construction method for swap-invariant sequences, which consists in a multiplication of a given swap-invariant or exchangeable sequence by a random factor and a simultaneous change of the probability measure. Then we prove that two large classes of swap-invariant sequences can always be represented in this way, namely by using an ergodic limit as the random factor; in Theorem 2.6 we use the ergodic limit of the sequence itself, in Theorem 2.17 the limit of $p$-norms of the means. The proofs are based on the results of Molchanov et al. (2014); Hardin (1981).

In Section 3 our results are extended to random measures on $[0,1]$ and $\mathbb{R}_{+}$. Exchangeable random measures are well known, see for example Kallenberg (1983), Chapter 9; Kallenberg (2002), Chapter 12; Kallenberg (2005). Our definition of swap-invariance is strictly weaker than exchangeability. More precisely, if a random measure $\xi$ is exchangeable, then the vector $\left(\xi\left(A_{1}\right), \ldots, \xi\left(A_{n}\right)\right)$ is exchangeable for all disjoint $A_{j}$ with equal Lebesgue measure; if $\xi$ is swap-invariant, then this vector is merely swap-invariant.

Similar to the case of sequences we provide a construction method for swapinvariant random measures, which is based on a change of the probability measure. We give an example of a swap-invariant non-exchangeable point process derived from a Poisson process. It is demonstrated that a swap-invariant random measure on $[0,1]$ can be represented as $\xi=\xi([0,1]) \eta$ where $\eta$ is exchangeable under a certain probability measure. Finally the ergodic theorem for swap-invariant random measures is shown, that is $\xi\left(A_{n}\right) / \lambda\left(A_{n}\right) \rightarrow X$ almost surely as $n \rightarrow \infty$ for some integrable random variable $X$. Here $\left(A_{n}\right)_{n \geq 1}$ is any increasing sequence of measurable sets such that $\lambda\left(A_{n}\right)<\infty$ and $\lambda\left(A_{n}\right) \rightarrow \infty$ as $n \rightarrow \infty$. The ergodic limit always exists, and it is independent of the sequence of sets under certain assumptions. As a consequence we obtain the representation

$$
\xi=X \eta
$$

where the random measure $\eta$ is exchangeable under a certain probability measure. A variant of this theorem is proven for the special case of exchangeable random measures.

\section{Swap-invariant sequences}

2.1. Sequences with finitely many values.

Lemma 2.1. Let $d \geq 1$ and $\xi$ and $\xi^{*}$ be integrable random vectors in $\mathbb{R}^{d}$. If $\xi$ and $\xi^{*}$ are $z$ onoid equivalent, then also the vectors $\left(\left|\xi_{1}\right|, \ldots,\left|\xi_{d}\right|\right)$ and $\left(\left|\xi_{1}^{*}\right|, \ldots,\left|\xi_{d}^{*}\right|\right)$ are zonoid equivalent. 
Proof: Note that for each $u \in \mathbb{R}^{d}$ the function $f(x)=\left|\sum_{j=1}^{d} u_{j}\right| x_{j}||$ on $\mathbb{R}^{d}$ is measurable, even, and positively homogeneous. By Molchanov et al. (2014), Theorem 2, we obtain $\mathbf{E} f(\xi)=\mathbf{E} f\left(\xi^{*}\right)$ for such $f$, which proves the assertion.

For $n \geq 1$ we write $\operatorname{Perm}(n)$ for the family of permutations of $\{1, \ldots, n\}$.

Theorem 2.2. Let $\xi$ be a swap-invariant random vector whose coordinates take only values $a$ and $b$ with $|a| \neq|b|$. Then $\xi$ is exchangeable.

Proof: First assume that $0 \leq a<b$. Fix $n \geq 2$. For $1 \leq d \leq n$ we denote by $N_{d}$ the set of vectors $k \in\{1, \ldots, n\}^{d}$ such that $1 \leq k_{1}<k_{2}<\ldots<k_{d}$. Furthermore we define $N=\bigcup_{1 \leq d \leq n} N_{d}$. For $k \in N$ the dimension of the vector $k$ is denoted by $|k|$. For a random vector $\xi$ in $\{a, b\}^{n}$ define

$$
z_{\xi}(k)=\mathbf{E} \max \left\{\xi_{k_{1}}, \ldots, \xi_{k_{|k|}}\right\}, k \in N .
$$

We first prove that the distribution of $\xi$ is uniquely determined by $z_{\xi}$ and then show that $z_{\xi}$ is invariant under permutations of $\xi$ if $\xi$ is swap-invariant. The marginal distributions of $\xi$ are denoted as follows:

$$
p(k ; m)=\mathbf{P}\left(\xi_{k_{1}}=m_{1}, \ldots, \xi_{k_{d}}=m_{d}\right)
$$

where $1 \leq d \leq n, k \in N_{d}$, and $m \in\{a, b\}^{d}$. Then, for $k \in N$,

$$
p(k ;(a, \ldots, a))=\frac{b-z_{\xi}(k)}{b-a} .
$$

We show that all marginal probabilities are functions of $z_{\xi}$. This is obvious for $k \in N_{1}$ since (2.1) yields

$$
p\left(k_{1} ; a\right)=\frac{b-z_{\xi}\left(k_{1}\right)}{b-a}, \quad \text { and } \quad p\left(k_{1} ; b\right)=1-p\left(k_{1} ; a\right) .
$$

Now let $1 \leq d \leq n-1$ and suppose that the probabilities $p(k ; m)$ are known for all $k \in N_{d}$ and $m \in\{a, b\}^{d}$. Fix $k \in N_{d+1}$. For $1 \leq j \leq d+1$ and $m \in\{a, b\}^{d+1}$, we obtain by summation over the $j$ th coordinate that

$$
\begin{aligned}
& p\left(\left(k_{1}, \ldots, k_{j-1}, k_{j+1}, \ldots, k_{d+1}\right) ;\left(m_{1}, \ldots, m_{j-1}, m_{j+1}, \ldots, m_{d+1}\right)\right)= \\
& \quad p\left(k ;\left(m_{1}, \ldots, m_{j-1}, a, m_{j+1}, \ldots, m_{d+1}\right)\right) \\
& \quad+p\left(k ;\left(m_{1}, \ldots, m_{j-1}, b, m_{j+1}, \ldots, m_{d+1}\right)\right) .
\end{aligned}
$$

By (2.1) and (2.2) all probabilities for the chosen $k$ can be calculated iteratively. This shows that all marginal probabilities are determined by $z_{\xi}$. Now let $\pi \in$ $\operatorname{Perm}(n)$. If $\xi$ is swap-invariant, then $z_{\xi}(k)=\mathbf{E} \max \left\{\xi_{1}, \ldots, \xi_{|k|}\right\}$ for $k \in N$ by Molchanov et al. (2014), Theorem 2, and therefore $z_{\xi}=z_{\xi \circ \pi}$. By the above argument $\xi$ and $\xi \circ \pi$ have the same distribution. Therefore $\xi$ is exchangeable.

To show the claim for general $a$ and $b$ note that the random vector $|\xi|$ is swapinvariant by Lemma 2.1, and hence it is exchangeable by the proof above. Since $|a| \neq|b|$, it follows that $\xi$ is exchangeable.

It is not difficult to construct a random sequence in $\{-1,+1\}$ that is swapinvariant but not exchangeable, e.g. using the method of Proposition 2.3. Moreover Example 2.5 provides a swap-invariant but non-exchangeable sequence whose coordinates take three values. 
2.2. Construction method. The next proposition provides a method to construct swap-invariant sequences from another swap-invariant sequence. It is a direct consequence of the definition of swap-invariance.

Proposition 2.3. Let $\eta$ be a sequence that is swap-invariant under a probability measure $\mathbf{Q}$. Further let $X$ be a random variable with $X \neq 0$ Q-almost surely and $c=\mathbf{E}_{\mathbf{Q}}\left[|X|^{-1}\right]<\infty$. Define another probability measure $\mathbf{P}$ by

$$
\frac{\mathrm{d} \mathbf{P}}{\mathrm{d} \mathbf{Q}}=\frac{1}{c|X|}
$$

Then the sequence $\xi_{j}=X \eta_{j}(j \geq 1)$ is swap-invariant under $\mathbf{P}$.

Note that in Proposition 2.3 we have $\mathbf{P} \sim \mathbf{Q}$. By Molchanov et al. (2014), Theorem 17, there exists a random variable $Y$ such that $\mathbf{E}_{\mathbf{Q}}|Y|<\infty$ and

$$
n^{-1} \sum_{j=1}^{n} \eta_{j} \rightarrow Y \quad \text { a.s. }
$$

Therefore also

$$
n^{-1} \sum_{j=1}^{n} \xi_{j} \rightarrow X Y \quad \text { a.s. }
$$

If the convergence (2.3) is in $L^{1}(\mathbf{Q})$ (which is the case, for example, if $\eta$ is exchangeable and integrable under $\mathbf{Q}$ ), then the convergence (2.4) is in $L^{1}(\mathbf{P})$.

Example 2.4. Let $\eta$ be a random sequence that is i.i.d. under $\mathbf{Q}$ with $\mathbf{E}_{\mathbf{Q}}\left|\eta_{1}\right|<\infty$ and $\eta_{1} \neq 0$ Q-almost surely. Set $X=\eta_{1}^{-1}$ in Proposition 2.3. Then the sequence $\xi_{1}=1, \xi_{j}=\eta_{j} / \eta_{1}(j \geq 2)$ is swap-invariant under $\mathbf{P}$. $\xi$ is not exchangeable under $\mathbf{P}$. For the exchangeability of $\xi$ under $\mathbf{P}$ would imply that $\xi_{2} \stackrel{d}{=} 1$ under $\mathbf{P}$ and $\mathbf{Q}$, whence $\eta_{1}=\eta_{2} \mathbf{Q}$-almost surely.

The following example shows that there exists a swap-invariant but not exchangeable sequence that takes only three values, in contrast to Theorem 2.2.

Example 2.5. Consider the special case of Example 2.4 where $\eta_{1}$ takes values 1 and 2 with probability $1 / 2$. We obtain the following finite-dimensional distributions of $\xi$ :

$$
\begin{aligned}
& \mathbf{P}\left(\xi_{2}=m_{2}, \ldots, \xi_{n}=m_{n}\right) \\
& \quad=\frac{2^{1-n}}{3}\left(\mathbb{1}\left\{m_{2}, \ldots, m_{n} \in\{1,2\}\right\}+2 \cdot \mathbb{1}\left\{m_{2}, \ldots, m_{n} \in\{1 / 2,1\}\right\}\right)
\end{aligned}
$$

where $n \geq 2$ and $m_{2}, \ldots, m_{n} \in\{1 / 2,1,2\}$. An explicit calculation yields

$$
\mathbf{E}_{\mathbf{P}}\left|\sum_{j=1}^{n} u_{j} \xi_{j}\right|=\frac{2^{1-n}}{3} \sum_{m_{1}, \ldots, m_{n} \in\{1,2\}}\left|\sum_{j=1}^{n} u_{j} m_{j}\right| .
$$

So $\xi$ is swap-invariant under $\mathbf{P}$.

2.3. Ergodic representation. As stated in Molchanov et al. (2014), Theorem 17, for each swap-invariant sequence, the mean converges almost surely to an integrable random variable. We now demonstrate that, if the ergodic limit is different from zero and if the convergence is in $L^{1}$, the limit can be used to characterize swapinvariant sequences as scaled exchangeable sequences under another probability measure. Thereby again the result of Hardin (1981) is applied. 
Theorem 2.6. Let $\xi$ be a random sequence that is swap-invariant under a probability measure $\mathbf{P}$ such that $n^{-1} \sum_{j=1}^{n} \xi_{j} \rightarrow X \mathbf{P}$-almost surely and in $L^{1}(\mathbf{P})$ as $n \rightarrow \infty$ with $\mathbf{P}(X \neq 0)=1$. Then there exists a random sequence $\eta$ that is exchangeable and integrable under the probability measure $\mathbf{Q}$ defined by

$$
\frac{\mathrm{d} \mathbf{Q}}{\mathrm{d} \mathbf{P}}=\frac{|X|}{\mathbf{E}_{\mathbf{P}}|X|}
$$

such that $\xi=X \eta \mathbf{P}$-almost surely.

Proof: Define

$$
\eta_{j}=\left\{\begin{array}{ll}
\xi_{j} / X & \text { on }\{X \neq 0\} \\
0 & \text { on }\{X=0\}
\end{array}, \quad j \geq 1 .\right.
$$

For $j \geq 1, \mathbf{E}_{\mathbf{P}}\left|\xi_{j}\right|<\infty$ implies $\mathbf{E}_{\mathbf{Q}}\left|\eta_{j}\right|<\infty$. Let $n \geq 1, u \in \mathbb{R}^{n}$, and $\pi \in \operatorname{Perm}(n)$. For $m \geq n$, the swap-invariance of $\xi$ under $\mathbf{P}$ yields

$$
\begin{aligned}
\mathbf{E}_{\mathbf{P}}\left|m^{-1} \sum_{k=1}^{m} \xi_{k}+\sum_{j=1}^{n} u_{j} \xi_{j}\right| & =\mathbf{E}_{\mathbf{P}}\left|m^{-1} \sum_{k=n+1}^{m} \xi_{k}+\sum_{j=1}^{n}\left(u_{j}+m^{-1}\right) \xi_{j}\right| \\
& =\mathbf{E}_{\mathbf{P}}\left|m^{-1} \sum_{k=n+1}^{m} \xi_{k}+\sum_{j=1}^{n}\left(u_{\pi(j)}+m^{-1}\right) \xi_{j}\right| \\
& =\mathbf{E}_{\mathbf{P}}\left|m^{-1} \sum_{k=1}^{m} \xi_{k}+\sum_{j=1}^{n} u_{\pi(j)} \xi_{j}\right| .
\end{aligned}
$$

Letting $m \rightarrow \infty$ we obtain

$$
\mathbf{E}_{\mathbf{P}}\left|X+\sum_{j=1}^{n} u_{j} \xi_{j}\right|=\mathbf{E}_{\mathbf{P}}\left|X+\sum_{j=1}^{n} u_{\pi(j)} \xi_{j}\right| .
$$

After change of measure this gives $\mathbf{E}_{\mathbf{Q}}\left|1+\sum_{j=1}^{n} u_{j} \eta_{j}\right|=\mathbf{E}_{\mathbf{Q}}\left|1+\sum_{j=1}^{n} u_{\pi(j)} \eta_{j}\right|$. Since this holds for all $u \in \mathbb{R}^{n}$, it follows by Hardin (1981), Theorem 1.1, that $\eta$ is exchangeable under $\mathbf{Q}$.

Example 2.7. Let $\left(Z_{j}\right)_{j \geq 1}$ be i.i.d. standard normal random variables under a probability measure $\mathbf{P}$, and let $\left(b_{j}\right)_{j \geq 1}$ be a sequence of real numbers such that $\beta=\sum_{j \geq 1} b_{j}^{2}<\infty$. Define a random sequence $(\xi)_{j \geq 1}$ by $\xi_{j}=\exp \zeta_{j}$ where

$$
\zeta_{j}=Z_{j}+\sum_{k=1}^{\infty} b_{k} Z_{k}+\mu_{j}, \quad \mu_{j}=-\frac{1}{2}\left(1+\beta+2 b_{j}\right) .
$$

Note that $\xi_{i} \stackrel{d}{=} \xi_{j}$ if and only if $b_{i}=b_{j}$. In Molchanov et al. (2014), Examples 15 and 25 , it is shown that $\xi$ is swap-invariant under $\mathbf{P}$ and that the limit

$$
X=\lim _{n \rightarrow \infty} n^{-1} \sum_{j=1}^{n} \xi_{j}=\exp \left(\sum_{k=1}^{\infty} b_{k} Z_{k}-\frac{1}{2} \beta\right)
$$

exists $\mathbf{P}$-almost surely and in $L^{1}(\mathbf{P})$. By Theorem 2.6 the random sequence

$$
\eta_{j}=\frac{\xi_{j}}{X}=\exp \left(Z_{j}-b_{j}-\frac{1}{2}\right)
$$

is exchangeable under $\mathbf{Q}$. 
In the context of Theorem 2.6 de Finetti's theorem implies a relation between $\xi_{1}$ and $X$ that we state in the following corollary. As usual the $\sigma$-algebra generated by the open sets in $\mathbb{R}^{\infty}$ is denoted by $\mathcal{B}\left(\mathbb{R}^{\infty}\right)$, the tail $\sigma$-algebra of $\mathcal{B}\left(\mathbb{R}^{\infty}\right)$ by $\mathcal{T}$, the shift-invariant $\sigma$-algebra by $\mathcal{I}$, and the $\sigma$-algebra of sets that are invariant under all finite permutations by $\mathcal{E}$. For a random sequence $\eta$ we define the corresponding $\sigma$-algebras on the basic probability space by

$$
\mathcal{T}_{\eta}=\eta^{-1} \mathcal{T}, \quad \mathcal{I}_{\eta}=\eta^{-1} \mathcal{I}, \quad \mathcal{E}_{\eta}=\eta^{-1} \mathcal{E} .
$$

It is well known that, if $\eta$ is exchangeable, then $\mathcal{T}_{\eta}=\mathcal{I}_{\eta}=\mathcal{E}_{\eta}$ almost surely, i.e. their completions are equal, see for example Kallenberg (2005), Corollary 1.6. The $\operatorname{sign}$ of $x \in \mathbb{R}$ is denoted by $\operatorname{sign}(x)$.

Corollary 2.8. Under the conditions of Theorem 2.6

$$
\mathbf{E}_{\mathbf{P}}\left[\operatorname{sign}(X) \xi_{j} \mid \mathcal{T}_{\eta}\right]=\mathbf{E}_{\mathbf{P}}\left[|X| \mid \mathcal{T}_{\eta}\right] \quad \text { a.s. }, j \geq 1,
$$

where $\eta$ is defined as in Theorem 2.6.

Proof: First note that $\mathbf{P}$ and $\mathbf{Q}$ are equivalent, so we may say that an equality or convergence holds 'almost surely' without specifying the probability measure. Now on the one hand the definition of $\eta$ implies that $n^{-1} \sum_{k=1}^{n} \eta_{k} \rightarrow 1$ almost surely as $n \rightarrow \infty$. On the other hand Kallenberg (2005), Theorem 1.1, yields

$$
n^{-1} \sum_{k=1}^{n} \eta_{k} \rightarrow \mathbf{E}_{\mathbf{Q}}\left[\eta_{j} \mid \mathcal{T}_{\eta}\right]=\frac{\mathbf{E}_{\mathbf{P}}\left[|X| \eta_{j} \mid \mathcal{T}_{\eta}\right]}{\mathbf{E}_{\mathbf{P}}\left[|X| \mid \mathcal{T}_{\eta}\right]}=\frac{\mathbf{E}_{\mathbf{P}}\left[\operatorname{sign}(X) \xi_{j} \mid \mathcal{T}_{\eta}\right]}{\mathbf{E}_{\mathbf{P}}\left[|X| \mid \mathcal{T}_{\eta}\right]} \quad \text { a.s. }, j \geq 1
$$

2.4. -norm representation. The connection between swap-invariant sequences and exchangeable sequences established in the preceding section is restricted to cases where the ergodic limit is attained in $L^{1}$ and is almost surely different from zero. Now we present another method, using $p$-norms, where the second condition can be dropped. However $L^{1}$-convergence is still required.

For a sequence $x \in \mathbb{R}^{\infty}$ the vector of the first $n$ components of $x$ is denoted by $x^{(n)}=\left(x_{1}, \ldots, x_{n}\right)$. We define, for $x \in \mathbb{R}^{\infty}, n \geq 1$, and $1 \leq p<\infty$,

$$
\|x\|_{p}^{(n)}=\left(n^{-1} \sum_{j=1}^{n}\left|x_{j}\right|^{p}\right)^{1 / p}, \quad\|x\|_{p}=\limsup _{n \rightarrow \infty}\|x\|_{p}^{(n)} \in \overline{\mathbb{R}}_{+},
$$

and

$$
\|x\|_{\infty}^{(n)}=\max \left\{\left|x_{j}\right| ; 1 \leq j \leq n\right\}, \quad\|x\|_{\infty}=\limsup _{n \rightarrow \infty}\|x\|_{\infty}^{(n)} \in \overline{\mathbb{R}}_{+} .
$$

For the proof of the main result in this section, Theorem 2.12, some preliminary results are required.

Proposition 2.9. Let $X, Y, X_{n}, Y_{n}(n \geq 1)$ be non-negative random variables such that $X_{n} \rightarrow X$ as $n \rightarrow \infty$ almost surely and in $L^{1}, Y_{n}$ and $Y$ are bounded by $K$ for some $K>0$, and $Y_{n} \rightarrow Y$ almost surely on the event $\{X>0\}$. Then $X_{n} Y_{n} \rightarrow X Y$ almost surely and in $L^{1}$.

Proof: We have

$$
\begin{aligned}
\mathbf{E}\left|X_{n} Y_{n}-X Y\right|= & \mathbf{E}\left|\left(X_{n}-X\right)\left(Y_{n}-Y\right)\right|+\mathbf{E}\left|X\left(Y_{n}-Y\right)\right|+\mathbf{E}\left|\left(X_{n}-X\right) Y\right| \\
\leq & 2 K \mathbf{E}\left|X_{n}-X\right|+\mathbf{E}\left[\left|X\left(Y_{n}-Y\right)\right| \mathbb{1}\{X>0\}\right] \\
& +K \mathbf{E}\left|X_{n}-X\right| .
\end{aligned}
$$


All three terms on the right-hand side converge to zero as $n \rightarrow \infty$.

The following lemma is a consequence of Molchanov et al. (2014), Theorem 2, and Kallenberg (2002), Lemma 1.35.

Lemma 2.10. Let $\|\cdot\|$ be a norm on $\mathbb{R}^{d}$ where $d \geq 1$, and $\xi_{1}$, $\xi_{2}$ two random vectors in $\mathbb{R}^{d}$ that are integrable under a probability measure $\mathbf{P}$, and either both symmetric or both supported by $\mathbb{R}_{+}^{d}$. For $i \in\{1,2\}$ assume $\mathbf{E}_{\mathbf{P}}\left\|\xi_{i}\right\|>0$ and define a probability measure $\mathbf{Q}_{i}$ by

$$
\frac{\mathrm{d} \mathbf{Q}_{i}}{\mathrm{~d} \mathbf{P}}=\frac{\left\|\xi_{i}\right\|}{\mathbf{E} \mathbf{P}\left\|\xi_{i}\right\|} .
$$

If $\xi_{1}$ and $\xi_{2}$ are zonoid equivalent under $\mathbf{P}$, then $\mathbf{E}_{\mathbf{P}}\left\|\xi_{1}\right\|=\mathbf{E}_{\mathbf{P}}\left\|\xi_{2}\right\|$ and

$$
\mathbf{Q}_{1}\left(\xi_{1} /\left\|\xi_{1}\right\| \in A\right)=\mathbf{Q}_{2}\left(\xi_{2} /\left\|\xi_{2}\right\| \in A\right), \quad A \in \mathcal{B}\left(\mathbb{R}^{d}\right) .
$$

Lemma 2.11. Fix $p \in[1, \infty]$. Let $\xi_{1}, \xi_{2}$ be two symmetric sequences of random variables such that $\xi_{1}^{(n)}$ and $\xi_{2}^{(n)}$ are zonoid equivalent under $\mathbf{P}$ for each $n \geq 1$, and

$$
\left\|\xi_{i}\right\|_{p}^{(n)} \rightarrow\left\|\xi_{i}\right\|_{p} \quad \mathbf{P} \text {-a.s. and in } L^{1}(\mathbf{P}) \text { as } n \rightarrow \infty \text {. }
$$

Then $\mathbf{E}_{\mathbf{P}}\left\|\xi_{1}\right\|_{p}=\mathbf{E}_{\mathbf{P}}\left\|\xi_{2}\right\|_{p}$, and if $\mathbf{E}_{\mathbf{P}}\left\|\xi_{1}\right\|_{p}>0$, the probability measures $\mathbf{Q}_{i}$ defined by

$$
\frac{\mathrm{d} \mathbf{Q}_{i}}{\mathrm{~d} \mathbf{P}}=\frac{\left\|\xi_{i}\right\|_{p}}{\mathbf{E}_{\mathbf{P}}\left\|\xi_{i}\right\|_{p}}, \quad i=1,2,
$$

satisfy $\mathbf{Q}_{1}\left(\xi_{1} /\left\|\xi_{1}\right\|_{p} \in B\right)=\mathbf{Q}_{2}\left(\xi_{2} /\left\|\xi_{2}\right\|_{p} \in B\right)$ for all $B \in \mathcal{B}\left(\mathbb{R}^{\infty}\right)$.

Proof: To simplify notation we omit the subscript $p$ at $\|x\|_{p}^{(n)}$ and $\|x\|_{p}$ for $x \in \mathbb{R}^{\infty}$ and $n \geq 1$ throughout the proof.

Let $n \geq 1$. Since $\xi_{1}^{(n)}$ and $\xi_{2}^{(n)}$ are zonoid equivalent, Lemma 2.10 implies that $\mathbf{E}_{\mathbf{P}}\left\|\xi_{1}\right\|^{(n)}=\mathbf{E}_{\mathbf{P}}\left\|\xi_{2}\right\|^{(n)}$. Letting $n \rightarrow \infty$ yields $\mathbf{E}_{\mathbf{P}}\left\|\xi_{1}\right\|=\mathbf{E}_{\mathbf{P}}\left\|\xi_{2}\right\|$.

Now assume $\mathbf{E}_{\mathbf{P}}\left\|\xi_{1}\right\|>0$. Choose $N \geq 1$ such that $\mathbf{E}_{\mathbf{P}}\left\|\xi_{1}\right\|^{(n)}>0$ for $n \geq N$. For $n \geq N$ and $i \in\{1,2\}$ define probability measures $\mathbf{Q}_{i}^{(n)}$ by

$$
\frac{\mathrm{d} \mathbf{Q}_{i}^{(n)}}{\mathrm{d} \mathbf{P}}=\frac{\left\|\xi_{i}\right\|^{(n)}}{\mathbf{E}_{\mathbf{P}}\left\|\xi_{i}\right\|^{(n)}} .
$$

By Lemma 2.10

$$
\mathbf{Q}_{1}^{(n)}\left(\xi_{1}^{(n)} /\left\|\xi_{1}\right\|^{(n)} \in A\right)=\mathbf{Q}_{2}^{(n)}\left(\xi_{2}^{(n)} /\left\|\xi_{2}\right\|^{(n)} \in A\right)
$$

for $A \in \mathcal{B}\left(\mathbb{R}^{n}\right)$. It follows that, for $n \geq N, 1 \leq k \leq n$, and $A \in \mathcal{B}\left(\mathbb{R}^{k}\right)$,

$$
\begin{aligned}
\mathbf{Q}_{1}^{(n)}\left(\frac{\xi_{1}^{(k)}}{\left\|\xi_{1}\right\|^{(n)}} \in A\right) & =\mathbf{Q}_{1}^{(n)}\left(\frac{\xi_{1}^{(n)}}{\left\|\xi_{1}\right\|^{(n)}} \in A \times \mathbb{R}^{n-k}\right) \\
& =\mathbf{Q}_{2}^{(n)}\left(\frac{\xi_{2}^{(n)}}{\left\|\xi_{2}\right\|^{(n)}} \in A \times \mathbb{R}^{n-k}\right)=\mathbf{Q}_{2}^{(n)}\left(\frac{\xi_{2}^{(k)}}{\left\|\xi_{2}\right\|^{(n)}} \in A\right) .
\end{aligned}
$$

Now let $f$ be a bounded continuous function from $\mathbb{R}^{k}$ to $\mathbb{R}_{+}$. Then, for $i \in\{1,2\}$,

$$
\mathbf{E}_{i}^{(n)} f\left(\frac{\xi_{i}^{(k)}}{\left\|\xi_{i}\right\|^{(n)}}\right)=\frac{1}{\mathbf{E}_{\mathbf{P}}\left\|\xi_{i}\right\|^{(n)}} \int_{\left\{\left\|\xi_{i}\right\|^{(n)}>0\right\}}\left\|\xi_{i}\right\|^{(n)} f\left(\frac{\xi_{i}^{(k)}}{\left\|\xi_{i}\right\|^{(n)}}\right) d \mathbf{P}
$$


where $\mathbf{E}_{i}^{(n)}$ denotes the expectation with respect to $\mathbf{Q}_{i}^{(n)}$. Now we apply Proposition 2.9 to the random variables

$$
\begin{aligned}
& X_{n}=\left\|\xi_{i}\right\|^{(n)}, \quad X=\left\|\xi_{i}\right\|, \\
& Y_{n}=\left\{\begin{array}{ll}
f\left(\frac{\xi_{i}^{(k)}}{\left\|\xi_{i}\right\|^{(n)}}\right) & \text { on }\left\{\left\|\xi_{i}\right\|^{(n)}>0\right\} \\
0 & \text { on }\left\{\left\|\xi_{i}\right\|^{(n)}=0\right\}
\end{array},\right. \\
& Y= \begin{cases}f\left(\frac{\xi_{i}^{(k)}}{\left\|\xi_{i}\right\|}\right) & \text { on }\left\{\left\|\xi_{i}\right\|>0\right\} \\
0 & \text { on }\left\{\left\|\xi_{i}\right\|=0\right\}\end{cases}
\end{aligned}
$$

In particular, note that for a point $\omega$ with $\left\|\xi_{i}(\omega)\right\|>0$ we have $\left\|\xi_{i}(\omega)\right\|^{(n)}>0$ for all sufficiently large $n$. Hence the continuity of $f$ implies that $Y_{n} \rightarrow Y$ as $n \rightarrow \infty$ $\mathbf{P}$-almost surely on the event $\left\{\left\|\xi_{i}\right\|>0\right\}$. We conclude that the right-hand side of (2.6) converges to

$$
\frac{1}{\mathbf{E}_{\mathbf{P}}\left\|\xi_{i}\right\|} \int_{\left\{\left\|\xi_{i}\right\|>0\right\}}\left\|\xi_{i}\right\| f\left(\frac{\xi_{i}^{(k)}}{\left\|\xi_{i}\right\|}\right) d \mathbf{P}=\mathbf{E}_{i} f\left(\frac{\xi_{i}^{(k)}}{\left\|\xi_{i}\right\|}\right)
$$

where $\mathbf{E}_{i}$ denotes the expectation with respect to $\mathbf{Q}_{i}$. Using equality of the distributions in (2.5) yields $\mathbf{E}_{1} f\left(\xi_{1}^{(k)} /\left\|\xi_{1}\right\|\right)=\mathbf{E}_{2} f\left(\xi_{2}^{(k)} /\left\|\xi_{2}\right\|\right)$. By approximation it follows that $\mathbf{Q}_{1}\left(\xi_{1}^{(k)} /\left\|\xi_{1}\right\| \in A\right)=\mathbf{Q}_{2}\left(\xi_{2}^{(k)} /\left\|\xi_{2}\right\| \in A\right)$ for each $A \in \mathcal{B}\left(\mathbb{R}^{k}\right)$. Since this holds for all $k \geq 1$, we obtain $\mathbf{Q}_{1}\left(\xi_{1} /\left\|\xi_{1}\right\| \in B\right)=\mathbf{Q}_{2}\left(\xi_{2} /\left\|\xi_{2}\right\| \in B\right)$ for each $B \in \mathcal{B}\left(\mathbb{R}^{\infty}\right)$.

Theorem 2.12. Fix $p \in[1, \infty]$. Let $\xi$ be a random sequence that is swap-invariant under a probability measure $\mathbf{P}$ such that $\|\xi\|_{p}^{(n)} \rightarrow\|\xi\|_{p}$ as $n \rightarrow \infty \mathbf{P}$-almost surely and in $L^{1}(\mathbf{P})$, and $\mathbf{E}_{\mathbf{P}}\|\xi\|_{p}>0$. Define another probability measure $\mathbf{Q}$ by

$$
\frac{\mathrm{d} \mathbf{Q}}{\mathrm{d} \mathbf{P}}=\frac{\|\xi\|_{p}}{\mathbf{E}_{\mathbf{P}}\|\xi\|_{p}} .
$$

(i) There exists a random sequence $\zeta$ that is exchangeable under $\mathbf{Q}$ such that

$$
\varepsilon \xi_{j}=\|\xi\|_{p} \zeta_{j} \quad \mathbf{P} \text {-a.s. }, j \geq 1
$$

where $\varepsilon$ is a random variable that takes values \pm 1 with probabilities $1 / 2$ and is independent of $\xi$ under $\mathbf{P}$.

(ii) If $\xi$ is either symmetric or non-negative, then there exists a random sequence $\eta$ that is exchangeable under $\mathbf{Q}$ such that

$$
\xi_{j}=\|\xi\|_{p} \eta_{j} \quad \mathbf{P} \text {-a.s. }, j \geq 1 .
$$

Proof: To simplify notation we again omit the subscript $p$ at $\|x\|_{p}^{(n)}$ and $\|x\|_{p}$ for $x \in \mathbb{R}^{\infty}$ and $n \geq 1$.

First assume that $\xi$ is symmetric under $\mathbf{P}$. Let $d \geq 1$ and $\pi \in \operatorname{Perm}(d)$, and denote by $\xi \circ \pi$ the random sequence that results from $\xi$ by applying the permutation $\pi$ to the first $d$ members. Then $\|\xi\|=\|\xi \circ \pi\|$. Moreover $\xi^{(n)}$ and $(\xi \circ \pi)^{(n)}$ 
are zonoid equivalent under $\mathbf{P}$ for all $n \geq 1$. Define

$$
\eta_{j}=\left\{\begin{array}{ll}
\frac{\xi_{j}}{\|\xi\|} & \text { on }\{\|\xi\|>0\} \\
0 & \text { on }\{\|\xi\|=0\}
\end{array} \quad, \quad j \geq 1 .\right.
$$

By Lemma 2.11

$$
\mathbf{Q}(\eta \in B)=\mathbf{Q}\left(\frac{\xi}{\|\xi\|} \in B\right)=\mathbf{Q}\left(\frac{\xi \circ \pi}{\|\xi\|} \in B\right)=\mathbf{Q}(\eta \circ \pi \in B) .
$$

for all $B \in \mathcal{B}\left(\mathbb{R}^{\infty}\right)$. This shows that $\eta$ is exchangeable under $\mathbf{Q}$. It remains to show that $\xi_{j}=0 \mathbf{P}$-almost surely on the event $\{\|\xi\|=0\}$ for all $j \geq 1$. Define random sequences $\xi^{>}$and $\xi^{=}$by

$$
\xi_{j}^{>}=\xi_{j} \mathbb{1}\{\|\xi\|>0\}, \quad \xi_{j}^{=}=\xi_{j} \mathbb{1}\{\|\xi\|=0\}
$$

for $j \geq 1$. For $n \geq 1$ and $u \in \mathbb{R}^{n}$ we have

$$
\begin{aligned}
\mathbf{E}_{\mathbf{P}}\left|\sum_{j=1}^{n} u_{j} \xi_{j}\right| & =\mathbf{E}_{\mathbf{P}} \mathbb{1}\{\|\xi\|>0\}\left|\sum_{j=1}^{n} u_{j} \xi_{j}\right| \\
& =\mathbf{E}_{\mathbf{P}}\|\xi\|\left|\sum_{j=1}^{n} u_{j} \eta_{j}\right|=\mathbf{E}_{\mathbf{P}}\|\xi\| \mathbf{E}_{\mathbf{Q}}\left|\sum_{j=1}^{n} u_{j} \eta_{j}\right|
\end{aligned}
$$

Hence $\xi^{>}$is swap-invariant under $\mathbf{P}$. This implies that also $\xi^{=}$is swap-invariant under $\mathbf{P}$. Since $\|\xi\|^{(n)} \rightarrow\|\xi\| \mathbf{P}$-almost surely and in $L^{1}(\mathbf{P})$,

$$
\left\|\xi^{=}\right\|^{(n)} \rightarrow\|\xi\| \mathbb{1}\{\|\xi\|=0\}=0 \quad \mathbf{P} \text {-a.s. and in } L^{1}(\mathbf{P}) .
$$

Since $\left\|\xi^{=}\right\|_{1}^{(n)} \leq\left\|\xi^{=}\right\|^{(n)}$ by the Hölder inequality, we conclude that $\left\|\xi^{=}\right\|_{1}^{(n)} \rightarrow 0$ Palmost surely and in $L^{1}(\mathbf{P})$. The swap-invariance of $\xi^{=}$implies that $\mathbf{E}_{\mathbf{P}}\left\|\xi^{=}\right\|_{1}^{(n)}=$ $\mathbf{E}_{\mathbf{P}}\left|\xi_{1}^{=}\right|$for all $n \geq 1$. Therefore $\mathbf{E}_{\mathbf{P}}\left|\xi_{j}\right|=\mathbf{E}_{\mathbf{P}}\left|\xi_{1}\right|=0$, hence $\xi_{j}^{\overline{=}}=0$ P-almost surely.

If $\xi$ is not symmetric, we may define a random sequence $\rho$ by $\rho_{j}=\varepsilon \xi_{j}$ for $j \geq 1$ where $\varepsilon$ has the stated properties. Then $\rho$ is symmetric and swap-invariant under $\mathbf{P}$. Applying the preceding proof to $\rho$ proves (i). In particular, if $\xi$ is non-negative, then also the sequence $\left(\left|\zeta_{1}\right|,\left|\zeta_{2}\right|, \ldots\right)$ is exchangeable under $\mathbf{Q}$, which shows the second statement of (ii).

In the following example a symmetric random sequence $\xi$ is defined that is swapinvariant but not exchangeable. Since each component as well as the ergodic limit is zero with positive probability, neither Molchanov et al. (2014), Theorem 21, nor Theorem 2.6 can be used to obtain a representation in terms of an exchangeable sequence. However Theorem 2.12 can be applied.

Example 2.13. Let $\rho$ be a random sequence that is i.i.d. under a probability measure $\mathbf{R}$ such that $\rho_{1}$ takes values $-1,0,+1$ with equal probability $1 / 3$. Further let $X=1+\left|\rho_{1}\right|$. Define another probability measure $\mathbf{P}$ by

$$
\frac{\mathrm{d} \mathbf{P}}{\mathrm{d} \mathbf{R}}=\frac{3}{2} X^{-1}
$$


and a random sequence $\xi_{j}=X \rho_{j}(j \geq 1)$. Then $\xi$ is swap-invariant under $\mathbf{P}$ by Proposition 2.3 and has finite-dimensional distributions

$$
\begin{aligned}
\mathbf{P}\left(\xi_{1}=m_{1}, \ldots, \xi_{n}=m_{n}\right) & \\
=3^{1-n} \cdot( & \frac{1}{2} \mathbb{1}\left\{m_{1}=0, m_{2}, \ldots, m_{n} \in\{-1,0,1\}\right\} \\
& \left.+\frac{1}{4} \mathbb{1}\left\{m_{1} \in\{-2,2\}, m_{2}, \ldots, m_{n} \in\{-2,0,2\}\right\}\right)
\end{aligned}
$$

where $n \geq 1$ and $m_{1}, \ldots, m_{n} \in\{-2,-1,0,1,2\}$. In particular,

$$
\mathbf{P}\left(\xi_{1}=0\right)=\frac{1}{2}, \quad \mathbf{P}\left(\xi_{1}= \pm 2\right)=\frac{1}{4},
$$

and, for $j \geq 2$,

$$
\mathbf{P}\left(\xi_{j}=0\right)=\frac{1}{3}, \quad \mathbf{P}\left(\xi_{j}= \pm 1\right)=\mathbf{P}\left(\xi_{j}= \pm 2\right)=\frac{1}{6} .
$$

Thus $\xi$ is symmetric and not exchangeable under $\mathbf{P}$. Since $\mathbf{P}\left(\xi_{j}=0\right)>0$ for all $j$, we cannot divide the sequence by one of its members in order to obtain an exchangeable sequence as done in Molchanov et al. (2014), Theorem 21. Further note that

$$
n^{-1} \sum_{j=1}^{n} \rho_{j} \rightarrow \mathbf{E}_{\mathbf{R}} \rho_{1}=0 \quad \text { R-a.s. }
$$

which implies $n^{-1} \sum_{j=1}^{n} \xi_{j} \rightarrow 0$ P-almost surely. Thus Theorem 2.6 is not applicable here either. In order to apply Theorem 2.12 fix $p=1$. Since the sequence $\left(\left|\rho_{j}\right|\right)_{j \geq 1}$ is i.i.d. under $\mathbf{R}$,

$$
n^{-1} \sum_{j=1}^{n}\left|\rho_{j}\right| \rightarrow \mathbf{E}_{\mathbf{R}}\left|\rho_{1}\right|=\frac{2}{3} \quad \text { R-a.s. and in } L^{1}(\mathbf{R}) .
$$

It follows that

$$
\|\xi\|_{1}^{(n)}=X n^{-1} \sum_{j=1}^{n}\left|\rho_{j}\right| \rightarrow \frac{2}{3} X=\|\xi\|_{1} \quad \text { P-a.s. and in } L^{1}(\mathbf{P})
$$

and $\mathbf{E}_{\mathbf{P}}\|\xi\|_{1}=1$. Thus the conditions of Theorem 2.12 are satisfied. We apply the definitions in Theorem 2.12,

$$
\frac{\mathrm{d} \mathbf{Q}}{\mathrm{d} \mathbf{P}}=\frac{2}{3} X, \quad \eta_{j}=\frac{3}{2} \rho_{j}, \quad j \geq 1,
$$

and find that $\mathbf{Q}=\mathbf{R}$. Theorem 2.12 says that $\eta$ is exchangeable under $\mathbf{Q}$, which can be immediately confirmed here.

An interesting special case of Theorem 2.12 is that of non-negative sequences and $p=1$. In this case the limit in Theorem 2.12 is the ordinary ergodic limit and the probability measure $\mathbf{Q}$ is defined as in Theorem 2.6. However the conditions are weaker than in Theorem 2.6 because the ergodic limit can be zero with positive P-probability here.

Example 2.14. The lognormal sequence in Example 2.7 satisfies the assumptions of Theorem 2.6, and therefore also those of Theorem 2.12 for $p=1$. 
A slightly more general case is $p=1$ and no sign restrictions on $\xi$. Application of Theorem 2.12 requires the mean of absolute values $n^{-1} \sum_{j=1}^{n}\left|\xi_{j}\right|$ to converge almost surely and in $L^{1}$. However if $\xi$ is swap-invariant, it follows from Lemma 2.1 that also the sequence of absolute values, $\left(\left|\xi_{1}\right|,\left|\xi_{2}\right|, \ldots\right)$, is swap-invariant, so the almost sure convergence is guaranteed by Molchanov et al. (2014), Theorem 17. Therefore only the $L^{1}$-convergence remains to be checked. By Scheffé's lemma, this reduces to the condition $\mathbf{E}\left|\xi_{1}\right|=\mathbf{E}\|\xi\|_{1}$. Also note that the inequality $\mathbf{E}\left|\xi_{1}\right| \geq \mathbf{E}\|\xi\|_{1}$ is always guaranteed by Fatou's lemma. We summarize the situation for the case $p=1$ as follows:

Proposition 2.15. Let $\xi$ be a swap-invariant random sequence. Then $n^{-1} \sum_{j=1}^{n}\left|\xi_{j}\right|$ converges almost surely to an integrable random variable $\|\xi\|_{1}$ as $n \rightarrow \infty$. If $\mathbf{E}\left|\xi_{1}\right|=\mathbf{E}\|\xi\|_{1}$, then this convergence is in $L^{1}$.

From Theorem 2.12 we finally derive a representation of the ergodic limit of symmetric or non-negative swap-invariant sequences. Again the general case is obtained by noting that, for a swap-invariant sequence $\xi$, the symmetric sequence $\varepsilon \xi$ is swap-invariant as well. In the derivation of Theorem 2.17 the formula for the conditional expectation under a change of the probability measure is used in the following form where the Radon-Nikodým derivative may be zero with non-zero probability.

Proposition 2.16. Let $(\Omega, \mathcal{F}, \mathbf{P})$ be a probability space, $\mathcal{G}$ a sub- $\sigma$-algebra of $\mathcal{F}, Z$ a random variable with $Z \geq 0$ and $\mathbf{E}_{\mathbf{P}} Z=1, \mathbf{Q}$ another probability measure defined by $\mathrm{d} \mathbf{Q} / \mathrm{d} \mathbf{P}=Z$, and $Y$ a random variable with $\mathbf{E}_{\mathbf{Q}}|Y|<\infty$. Then $\mathbf{E}_{\mathbf{P}}|Z Y|<\infty$, and

where

$$
\mathbb{1}\left\{Z_{0}>0\right\} \mathbf{E}_{\mathbf{Q}}[Y \mid \mathcal{G}]=\hat{Z}_{0} \mathbf{E}_{\mathbf{P}}[Z Y \mid \mathcal{G}] \quad \text { Q-a.s. }
$$

$$
Z_{0}=\mathbf{E}_{\mathbf{P}}[Z \mid \mathcal{G}], \quad \hat{Z}_{0}=\left\{\begin{array}{ll}
Z_{0}^{-1} & \text { on }\left\{Z_{0}>0\right\} \\
0 & \text { on }\left\{Z_{0}=0\right\}
\end{array} .\right.
$$

Theorem 2.17. Let $\xi$ be a symmetric or non-negative sequence of random variables that satisfies the conditions of Theorem 2.12 for some $p \in[1, \infty]$, and choose $\eta$ as in Theorem 2.12 (ii). Then

$$
n^{-1} \sum_{j=1}^{n} \xi_{j} \rightarrow\|\xi\|_{p} \hat{Y}_{0} \mathbf{E}_{\mathbf{P}}\left[\xi_{1} \mid \mathcal{T}_{\eta}\right] \quad \text { P-a.s. and in } L^{1}(\mathbf{P}) \text { as } n \rightarrow \infty,
$$

where

$$
Y_{0}=\mathbf{E}_{\mathbf{P}}\left[\|\xi\|_{p} \mid \mathcal{T}_{\eta}\right], \quad \hat{Y}_{0}=\left\{\begin{array}{ll}
Y_{0}^{-1} & \text { on }\left\{Y_{0}>0\right\} \\
0 & \text { on }\left\{Y_{0}=0\right\}
\end{array} .\right.
$$

Moreover,

$$
\left\{\|\xi\|_{p}>0\right\} \in \mathcal{T}_{\eta}, \quad \mathbf{P}\left(\left\{Y_{0}>0\right\} \Delta\left\{\|\xi\|_{p}>0\right\}\right)=0 .
$$

Proof: Since $\eta$ is $\mathbf{Q}$-integrable and exchangeable under $\mathbf{Q}$, it follows by Kallenberg (2002), Theorem 10.6, that

$$
n^{-1} \sum_{j=1}^{n} \eta_{j} \rightarrow \mathbf{E}_{\mathbf{Q}}\left[\eta_{1} \mid \mathcal{T}_{\eta}\right] \quad \text { Q-a.s. and in } L^{1}(\mathbf{Q}) .
$$


By Proposition 2.16,

$$
\mathbb{1}\left\{Y_{0}>0\right\} \mathbf{E}_{\mathbf{Q}}\left[\eta_{1} \mid \mathcal{T}_{\eta}\right]=\hat{Y}_{0} \mathbf{E}_{\mathbf{P}}\left[\xi_{1} \mid \mathcal{T}_{\eta}\right] \quad \text { Q-a.s. }
$$

We write $\|\xi\|$ for $\|\xi\|_{p}$ in the following. Multiplying both sides of (2.8) by $\|\xi\|$ shows that

$$
\mathbb{1}\left\{Y_{0}>0\right\} n^{-1} \sum_{j=1}^{n} \xi_{j} \rightarrow\|\xi\| \hat{Y}_{0} \mathbf{E}_{\mathbf{P}}\left[\xi_{1} \mid \mathcal{T}_{\eta}\right] \quad \text { Q-a.s. as } n \rightarrow \infty
$$

In order to see that the convergence (2.9) holds $\mathbf{P}$-almost surely, define $E=$ $\{\|\xi\|>0\}$. On $E^{c}$ we have $\xi_{j}=0 \mathbf{P}$-almost surely for all $j \geq 1$. On $E$ the measures $\mathbf{P}$ and $\mathbf{Q}$ are equivalent, so the convergence holds also $\mathbf{P}$-almost surely on $E$.

We now show that $E \in \mathcal{T}_{\eta}$. We may assume that $\eta_{j}=0$ on $E^{c}$ for $j \geq 1$. Define $A_{n}=\left\{\eta_{j}=0 ; j \geq n\right\}$ for $n \geq 1$. Note that

$$
A_{n} \subset\left\{\xi_{j}=0 ; j \geq n\right\} \subset E^{c},
$$

and $E^{c} \subset A_{n}$ by assumption. Hence $E^{c}=A_{n}$ for all $n \geq 1$, and therefore $E^{c} \in \mathcal{T}_{\eta}$.

We next show the last statement, which then implies that the convergence $(2.7)$ holds $\mathbf{P}$-almost surely. Now $\mathbf{E}_{\mathbf{P}}\left[Y_{0} \mathbb{1}_{A}\right]=\mathbf{E}_{\mathbf{P}}\left[\|\xi\| \mathbb{1}_{A}\right]$ for each $A \in \mathcal{T}_{\eta}$ by definition of conditional expectation. Define $F=\left\{Y_{0}>0\right\}$. Note that $\mathbf{E}_{\mathbf{P}}\left[Y_{0} \mathbb{1}_{A}\right]>0$ if and only if $\mathbf{P}(F \cap A)>0$, and $\mathbf{E}_{\mathbf{P}}\left[\|\xi\| \mathbb{1}_{A}\right]>0$ if and only if $\mathbf{P}(E \cap A)>0$. We conclude that $\mathbf{P}(F \cap A)>0$ if and only if $\mathbf{P}(E \cap A)>0$. It follows that $\mathbf{P}\left(F^{c} \backslash E^{c}\right)=$ $\mathbf{P}\left(F^{c} \cap E\right)=0$ because $F^{c} \in \mathcal{T}_{\eta}$. Moreover $\mathbf{P}\left(E^{c} \backslash F^{c}\right)=\mathbf{P}\left(E^{c} \cap F\right)=0$ because $E^{c} \in \mathcal{T}_{\eta}$. Thus $\mathbf{P}\left(F^{c} \Delta E^{c}\right)=0$.

To see that the convergence $(2.7)$ holds in $L^{1}(\mathbf{P})$ note that

$$
\mathbb{1}\left\{Y_{0}>0\right\}=\mathbb{1}\{\|\xi\|>0\}
$$

$\mathbf{P}$-almost surely and therefore also $\mathbf{Q}$-almost surely. It follows that

$$
\begin{aligned}
& \mathbf{E}_{\mathbf{P}}\left|n^{-1} \sum_{j=1}^{n} \xi_{j}-\|\xi\| \hat{Y}_{0} \mathbf{E}_{\mathbf{P}}\left[\xi_{1} \mid \mathcal{T}_{\eta}\right]\right| \\
& =\mathbf{E}_{\mathbf{P}}\|\xi\|\left|n^{-1} \sum_{j=1}^{n} \eta_{j}-\hat{Y}_{0} \mathbf{E}_{\mathbf{P}}\left[\xi_{1} \mid \mathcal{T}_{\eta}\right]\right| \\
& =\frac{1}{\mathbf{E}_{\mathbf{P}}\|\xi\|} \mathbf{E}_{\mathbf{Q}}\left|n^{-1} \sum_{j=1}^{n} \eta_{j}-\hat{Y}_{0} \mathbf{E}_{\mathbf{P}}\left[\xi_{1} \mid \mathcal{T}_{\eta}\right]\right| \\
& =\frac{1}{\mathbf{E}_{\mathbf{P}}\|\xi\|} \mathbf{E}_{\mathbf{Q}}\left|n^{-1} \sum_{j=1}^{n} \eta_{j}-\mathbb{1}\left\{Y_{0}>0\right\} \mathbf{E}_{\mathbf{Q}}\left[\eta_{1} \mid \mathcal{T}_{\eta}\right]\right| \\
& =\frac{1}{\mathbf{E}_{\mathbf{P}}\|\xi\|} \mathbf{E}_{\mathbf{Q}}\left|n^{-1} \sum_{j=1}^{n} \eta_{j}-\mathbb{1}\{\|\xi\|>0\} \mathbf{E}_{\mathbf{Q}}\left[\eta_{1} \mid \mathcal{T}_{\eta}\right]\right| \\
& =\frac{1}{\mathbf{E}_{\mathbf{P}}\|\xi\|} \mathbf{E}_{\mathbf{Q}}\left|n^{-1} \sum_{j=1}^{n} \eta_{j}-\mathbf{E}_{\mathbf{Q}}\left[\eta_{1} \mid \mathcal{T}_{\eta}\right]\right|
\end{aligned}
$$

The right-hand side converges to zero as $n \rightarrow \infty$. 
Theorem 2.17 says that under the stated conditions

$$
n^{-1} \sum_{j=1}^{n} \xi_{j} \rightarrow \frac{\|\xi\|_{p} \mathbf{E}_{\mathbf{P}}\left[\xi_{1} \mid \mathcal{T}_{\eta}\right]}{\mathbf{E}_{\mathbf{P}}\left[\|\xi\|_{p} \mid \mathcal{T}_{\eta}\right]} \quad \text { P-a.s. on }\left\{\|\xi\|_{p}>0\right\} \text { as } n \rightarrow \infty \text {. }
$$

In contrast to the representations of the ergodic limit in Theorem 21 and Proposition 22 in Molchanov et al. (2014), we may allow $\mathbf{P}\left(\xi_{j}=0\right)>0$ for all $j$ here. For non-negative $\xi$ and $p=1$, we summarize the results of Theorems 2.12 and 2.17 in the following corollary; note that this includes variants of Theorem 2.6 and Corollary 2.8.

Corollary 2.18. Let $\xi$ be a sequence of non-negative random variables that is swap-invariant under a probability measure $\mathbf{P}$ such that

$$
n^{-1} \sum_{j=1}^{n} \xi_{j} \rightarrow X \quad \mathbf{P} \text {-a.s. and in } L^{1}(\mathbf{P}) \text { as } n \rightarrow \infty
$$

with $\mathbf{E}_{\mathbf{P}} X>0$. Define the probability measure $\mathbf{Q}$ by

$$
\frac{\mathrm{d} \mathbf{Q}}{\mathrm{d} \mathbf{P}}=\frac{X}{\mathbf{E}_{\mathbf{P}} X} .
$$

Then there exists a random sequence $\eta$ that is exchangeable under $\mathbf{Q}$ such that $\xi_{j}=X \eta_{j} \mathbf{P}$-almost surely for $j \geq 1$. Moreover $\mathbf{E}_{\mathbf{P}}\left[X \mid \mathcal{T}_{\eta}\right]=\mathbf{E}_{\mathbf{P}}\left[\xi_{1} \mid \mathcal{T}_{\eta}\right] \mathbf{P}$-almost surely.

\section{Swap-invariant random measures}

3.1. Swap-invariance vs. exchangeability. Let $(\Omega, \mathcal{F}, \mathbf{P})$ be a probability space and $(S, \mathcal{S})$ a measurable space. A random measure $\xi$ on $S$ is a map $\xi: \Omega \times \mathcal{S} \rightarrow \overline{\mathbb{R}}_{+}$ such that $\xi(\cdot, M)$ is a $\overline{\mathbb{R}}_{+}$-valued random element for each $M \in \mathcal{S}$, and $\xi(\omega, \cdot)$ is a measure on $S$ for each $\omega \in \Omega$. The intensity measure of $\xi$ is $\mathbf{E} \xi(M)$ for $M \in \mathcal{S}$. A random measure $\xi$ on $S$ is called $\sigma$-finite if there exists a fixed measurable partition $\left(S_{j}\right)_{j \geq 1}$ of $S$ such that $\xi\left(S_{j}\right)<\infty$ almost surely for every $j \geq 1$. We only consider the cases $S=[0,1]$ and $\mathbb{R}_{+}$in this article, and $\mathcal{S}$ denotes the Borel $\sigma$-algebra $\mathcal{B}([0,1])$ or $\mathcal{B}\left(\mathbb{R}_{+}\right)$, respectively.

Definition 3.1. A random measure $\xi$ on $S=[0,1]$ or $\mathbb{R}_{+}$is called exchangeable if $\left(\xi\left(A_{1}\right), \ldots, \xi\left(A_{n}\right)\right) \stackrel{d}{=}\left(\xi\left(B_{1}\right), \ldots, \xi\left(B_{n}\right)\right)$ for any disjoint $A_{1}, \ldots, A_{n} \in \mathcal{S}, n \geq 1$, and any disjoint $B_{1}, \ldots, B_{n} \in \mathcal{S}$ with $\lambda\left(A_{j}\right)=\lambda\left(B_{j}\right)$ for $1 \leq j \leq n$.

Some equivalent formulations are given in Kallenberg (1983), Lemma 9.0. We investigate random measures that have a weaker property than exchangeability.

Definition 3.2. A random measure $\xi$ on $S=[0,1]$ or $\mathbb{R}_{+}$is called swap-invariant if $\mathbf{E}\left|\sum_{j=1}^{n} u_{j} \xi\left(A_{j}\right)\right|=\mathbf{E}\left|\sum_{j=1}^{n} u_{j} \xi\left(B_{j}\right)\right|$ for all $n \geq 1, u \in \mathbb{R}^{n}$, disjoint $A_{1}, \ldots, A_{n} \in$ $\mathcal{S}$, and disjoint $B_{1}, \ldots, B_{n} \in \mathcal{S}$ such that $\lambda\left(A_{j}\right)=\lambda\left(B_{j}\right), \mathbf{E} \xi\left(A_{j}\right)<\infty$, and $\mathbf{E} \xi\left(B_{j}\right)<\infty$ for $1 \leq j \leq n$.

Note that the integrands in Definition 3.2 are undefined for those points $\omega \in \Omega$ where two terms in the sum are infinite with opposite signs. However this can happen only with probability zero due to the integrability assumptions.

Several equivalent formulations of swap-invariance on a general measure space as well as their couterparts in the case of exchangeable random measures are given 
in Nagel (2016). Note that if $\xi$ is a swap-invariant random measure on $S$, then, for each $A \in \mathcal{S}$ with $\mathbf{E} \xi(A)<\infty, \lambda(A)=0$ implies $\xi(A)=0$ almost surely. Clearly, every exchangeable random measure is swap-invariant. Two more connections between the concepts of swap-invariance and exchangeability are now established. Proposition 3.3 provides a construction of swap-invariant random measures from a given swap-invariant random measure. In particular, one can use an exchangeable random measure to construct a swap-invariant random measure that is not exchangeable. In Theorem 3.5 we show how a swap-invariant random measure on $[0,1]$ can be expressed through an exchangeable random measure. In Section 3.2 a similar representation of swap-invariant random measures on $\mathbb{R}_{+}$is proven, which is based on the ergodic theorem and on the representation result for sequences derived in Section 2.

Proposition 3.3. Let $\eta$ be a random measure on $S=[0,1]$ or $\mathbb{R}_{+}$that is swapinvariant under a probability measure $\mathbf{Q}$. Further let $X$ be a random variable with $X>0 \quad$ Q-almost surely and $\mathbf{E}_{\mathbf{Q}}\left[X^{-1}\right]<\infty$, and $\mathbf{P}$ another probability measure defined by

$$
\frac{\mathrm{d} \mathbf{P}}{\mathrm{d} \mathbf{Q}}=\frac{1}{X \mathbf{E}_{\mathbf{Q}}\left[X^{-1}\right]} .
$$

Then the random measure $\xi$ defined by $\xi=X \eta$ is swap-invariant under $\mathbf{P}$.

Example 3.4. Let $\eta$ be a Poisson process on $[0,1]$ or $\mathbb{R}_{+}$under a probability measure $\mathbf{Q}$ with uniform intensity. Clearly $\eta$ is exchangeable under $\mathbf{Q}$. We may choose two disjoint measurable sets $K$ and $L$ with $\lambda(K)=\lambda(L)=1 / 2$. Define the random variable $X=1+\mathbb{1}\{\eta(K)>0\}$ and a new probability measure $\mathbf{P}$ by

$$
\frac{\mathrm{d} \mathbf{P}}{\mathrm{d} \mathbf{Q}}=\frac{1}{c X}, \quad c=\mathbf{E}_{\mathbf{Q}}\left[X^{-1}\right]=\frac{1}{2}\left(1+e^{-1 / 2}\right) .
$$

By Proposition 3.3 the point process $\xi=X \eta$ is swap-invariant under $\mathbf{P}$. We calculate

$$
\mathbf{P}(\xi(L)=0)=e^{-1 / 2} \neq \frac{2}{1+e^{1 / 2}}=\mathbf{P}(\xi(K)=0) .
$$

This shows that $\xi$ is not exchangeable under $\mathbf{P}$.

Theorem 3.5. Let $S=[0,1]$ and $\xi$ a random measure on $S$ that is swap-invariant under a probability measure $\mathbf{P}$ with $\mathbf{E}_{\mathbf{P}} \xi(S) \in(0, \infty)$. Then there exists a random measure $\eta$ that is exchangeable under the probability measure $\mathbf{Q}$ defined by

$$
\frac{\mathrm{d} \mathbf{Q}}{\mathrm{d} \mathbf{P}}=\frac{\xi(S)}{\mathbf{E}_{\mathbf{P}} \xi(S)}
$$

such that $\xi=\xi(S) \eta$.

Proof: Let $n \geq 1$ and $A_{j}, B_{j} \in \mathcal{S}$ with $\lambda\left(A_{j}\right)=\lambda\left(B_{j}\right)$ for $1 \leq j \leq n$ such that $\left(A_{j}\right)_{1 \leq j \leq n}$ are disjoint and $\left(B_{j}\right)_{1 \leq j \leq n}$ are disjoint. Define $A_{0}=S \backslash \bigcup_{j=1}^{n} A_{j}$ and $B_{0}=S \backslash \bigcup_{j=1}^{n} B_{j}$. Clearly, $\lambda\left(A_{0}\right)=\lambda\left(B_{0}\right)$. Now we define random vectors $\xi_{A}$ and $\xi_{B}$ in $\mathbb{R}_{+}^{n+1}$ by

$$
\xi_{A j}=\xi\left(A_{j}\right) \mathbb{1}\left\{\xi\left(A_{j}\right)<\infty\right\}, \quad \xi_{B j}=\xi\left(B_{j}\right) \mathbb{1}\left\{\xi\left(B_{j}\right)<\infty\right\}
$$

for $0 \leq j \leq n$. Here the indicator functions are $\mathbf{P}$-almost surely equal to 1 . Note that $\xi_{A}$ and $\xi_{B}$ are zonoid equivalent under $\mathbf{P}$. Moreover $\left\|\xi_{A}\right\|_{1}=\xi(S)=\left\|\xi_{B}\right\|_{1}$ 
$\mathbf{P}$-almost surely where $\|x\|_{1}=\sum_{j=0}^{n}\left|x_{j}\right|$ for $x \in \mathbb{R}^{n+1}$. Define

$$
\eta=\left\{\begin{array}{ll}
\frac{\xi}{\xi(S)} & \text { on }\{\xi(S)>0\} \\
0 & \text { on }\{\xi(S)=0\}
\end{array} .\right.
$$

Applying Lemma 2.10, it follows that, under $\mathbf{Q}$,

$$
\left(\eta\left(A_{1}\right), \ldots, \eta\left(A_{n}\right)\right) \stackrel{d}{=}\left(\eta\left(B_{1}\right), \ldots, \eta\left(B_{n}\right)\right)
$$

Hence $\eta$ is exchangeable under $\mathbf{Q}$.

Note that in the special case if $\xi$ is a random probability measure, i.e. $\xi([0,1])=1$, which is swap-invariant under $\mathbf{P}$, Theorem 3.5 says that $\xi$ is even exchangeable under $\mathbf{P}$.

Theorem 3.6. Let $\xi$ be a swap-invariant random measure on $S=[0,1]$ or $\mathbb{R}_{+}$ such that $\mathbf{E} \xi$ is $\sigma$-finite. Then $\mathbf{E} \xi=c \lambda$ for some $c \in \mathbb{R}_{+}$.

Proof: First assume that $S=[0,1]$ and $\mathbf{E} \xi(S)<\infty$. Then the claim follows from Theorem 3.5 and Kallenberg (2005), Theorem 1.25.

Now consider the case that either $S=[0,1]$ and $\mathbf{E} \xi(S)=\infty$, or $S=\mathbb{R}_{+}$and $\mathbf{E} \xi(S) \leq \infty$. Choose a measurable partition $\left(S_{j}\right)_{j \geq 1}$ of $S$ such that $\lambda\left(S_{j}\right)<\infty$ and $\mathbf{E} \xi\left(S_{j}\right)<\infty$ for all $j \geq 1$. Define $J=\left\{j \in \mathbb{Z}_{+} ; \lambda\left(S_{j}\right)>0\right\}$. Clearly $\xi\left(S_{j}\right)=0$ almost surely for $j \in \mathbb{Z}_{+} \backslash J$. By the first part of the proof

$$
\mathbf{E} \xi\left(S_{j} \cap A\right)=\frac{\mathbf{E} \xi\left(S_{j}\right)}{\lambda\left(S_{j}\right)} \lambda\left(S_{j} \cap A\right)
$$

for each $A \in \mathcal{S}$ and $j \in J$. If $|J| \geq 2$, let $i, j \in J$ with $i \neq j$ and consider the partition

$$
\mathcal{M}=\left\{S_{k} ; k \geq 1, k \neq i, k \neq j\right\} \cup\left\{S_{i} \cup S_{j}\right\}
$$

of $S$. Applying the previous result to the new partition $\mathcal{M}$ we obtain, in particular,

$\mathbf{E} \xi\left(S_{i}\right)=\mathbf{E} \xi\left(\left(S_{i} \cup S_{j}\right) \cap S_{i}\right)=\frac{\mathbf{E} \xi\left(S_{i} \cup S_{j}\right)}{\lambda\left(S_{i} \cup S_{j}\right)} \lambda\left(\left(S_{i} \cup S_{j}\right) \cap S_{i}\right)=\frac{\mathbf{E} \xi\left(S_{i} \cup S_{j}\right)}{\lambda\left(S_{i} \cup S_{j}\right)} \lambda\left(S_{i}\right)$,

and similarly for $i$ and $j$ interchanged. Consequently $c=\mathbf{E} \xi\left(S_{k}\right) / \lambda\left(S_{k}\right)$ for some $c \in \mathbb{R}_{+}$and all $k \in J$. Therefore

$$
\mathbf{E} \xi(A)=\sum_{j \in J} \mathbf{E} \xi\left(S_{j} \cap A\right)=c \sum_{j \in J} \lambda\left(S_{j} \cap A\right)=c \lambda(A)
$$

for each $A \in \mathcal{S}$.

Example 3.7. In Example 3.4 we have $\mathbf{E}_{\mathbf{Q}} \eta=\lambda$, which implies $\mathbf{E}_{\mathbf{P}} \xi=c^{-1} \lambda$.

By a similar proof it is shown in Nagel (2016), Theorem 2.19, that diffuse swapinvariant random measures can be represented as the randomly scaled Lebesgue measure. 
3.2. Ergodic representation. To formulate the ergodic theorem for swap-invariant random measures and the derived representation, we need to introduce some notions.

Definition 3.8. An increasing sequence $A_{n} \in \mathcal{B}\left(\mathbb{R}_{+}\right)$with $\lambda\left(A_{n}\right)<\infty$ for $n \geq 1$ and $\lambda\left(A_{n}\right) \rightarrow \infty$ as $n \rightarrow \infty$ is called standard sequence. For a standard sequence $\left(A_{n}\right)_{n \geq 1}$ we write

$$
\Delta A_{n}=\left\{\begin{array}{ll}
A_{1} & \text { if } n=1 \\
A_{n} \backslash A_{n-1} & \text { if } n \geq 2
\end{array} .\right.
$$

A standard sequence $\left(A_{n}\right)_{n \geq 1}$ is called standard sequence with constant increments if $\lambda\left(\Delta A_{n}\right)=c$ for all $n \geq 1$ and some $c \in(0, \infty)$. Moreover, given a random measure $\xi$ on $\mathbb{R}_{+}$, a standard sequence $\left(A_{n}\right)_{n \geq 1}$ is called $\xi$-integrable if $\mathbf{E} \xi\left(A_{n}\right)<\infty$ for $n \geq 1$.

For a fixed standard sequence with constant increments it is straightforward to derive the following ergodic theorem by applying the result for swap-invariant sequences Molchanov et al. (2014), Theorem 17.

Proposition 3.9. Let $\xi$ be a swap-invariant random measure on $\mathbb{R}_{+}$, and $\left(A_{n}\right)_{n \geq 1}$ a $\xi$-integrable standard sequence with constant increments. Then there exists an integrable random variable $X$ such that $\xi\left(A_{n}\right) / \lambda\left(A_{n}\right) \rightarrow X$ almost surely as $n \rightarrow \infty$.

Example 3.10. In Example 3.4 let $S=\mathbb{R}_{+}$. Further let $\left(A_{n}\right)_{n \geq 1}$ be a standard sequence with constant increments. Then $\eta\left(A_{n}\right) / \lambda\left(A_{n}\right) \rightarrow 1$ as $n \rightarrow \infty$ Q-almost surely and in $L^{1}(\mathbf{Q})$. It follows that $\xi\left(A_{n}\right) / \lambda\left(A_{n}\right) \rightarrow X \mathbf{Q}$-almost surely. Since $\mathbf{P}$ and $\mathbf{Q}$ are equivalent, this convergence holds also $\mathbf{P}$-almost surely. It can be shown by direct computation that the convergence is also in $L^{1}(\mathbf{P})$.

We show in Theorem 3.14 that the ergodic limit also exists if the increments are not necessarily constant and that the limit is unique under certain assumptions. This allows us to perform a change of the probability measure and to construct a random measure that is exchangeable under the new probability measure in order to obtain our representation (1.1). In the proof of Theorem 3.14 we make use of the following notion.

Definition 3.11. Let $\left(A_{n}\right)_{n \geq 1}$ be a standard sequence. A sequence $\left(C_{n}\right)_{n \geq 1}$ with constant increments is called compatible sequence with constant increments (CSCI) of $\left(A_{n}\right)$ if there exists a standard sequence $\left(B_{n}\right)_{n \geq 1}$ such that $\left(A_{n}\right)$ and $\left(C_{n}\right)$ are subsequences of $\left(B_{n}\right)$.

Obviously in this definition the sequences $\left(A_{n}\right),\left(B_{n}\right)$, and $\left(C_{n}\right)$ have the same limit set. We prove two lemmas on which Theorem 3.14 is based.

Lemma 3.12. Let $\left(A_{n}\right)_{n \geq 1}$ be a standard sequence, $\xi$ a random measure on $\mathbb{R}_{+}$, and $X$ a random variable.

(i) For each $c \in(0, \infty)$, there exists a CSCI $\left(C_{n}\right)$ of $\left(A_{n}\right)$ with $\lambda\left(C_{1}\right)=c$.

(ii) If $\xi\left(C_{n}\right) / \lambda\left(C_{n}\right) \rightarrow X$ almost surely as $n \rightarrow \infty$ for some $C S C I\left(C_{n}\right)$ of $\left(A_{n}\right)$, then also $\xi\left(A_{n}\right) / \lambda\left(A_{n}\right) \rightarrow X$ almost surely.

(iii) If $\xi\left(C_{n}\right) / \lambda\left(C_{n}\right) \rightarrow X$ in $L^{1}$ as $n \rightarrow \infty$ for some CSCI $\left(C_{n}\right)$ of $\left(A_{n}\right)$, then also $\xi\left(A_{n}\right) / \lambda\left(A_{n}\right) \rightarrow X$ in $L^{1}$.

Proof: (i) is clear because $\lambda$ is atomless. In order to prove (ii) and (iii) let $\left(C_{n}\right)$ be a CSCI of $\left(A_{n}\right)$. Define $c=\lambda\left(C_{1}\right)$ and $m_{k}=\min \left\{m \geq 1 ; A_{k} \subset C_{m}\right\}$ for $k \geq 1$. It follows that $1 \leq m_{1} \leq m_{2} \leq \ldots$, and $m_{k} \rightarrow \infty$ as $k \rightarrow \infty$. For large $k$ we 
have $m_{k} \geq 2$ and $C_{m(k)-1} \subset A_{k} \subset C_{m(k)}$ where the first inclusion is strict and the second may not. Hence, for large $k$,

$$
\lambda\left(C_{m(k)-1}\right) \leq \lambda\left(A_{k}\right) \leq \lambda\left(C_{m(k)}\right), \quad \xi\left(C_{m(k)-1}\right) \leq \xi\left(A_{k}\right) \leq \xi\left(C_{m(k)}\right),
$$

and therefore

$$
\begin{aligned}
\left(\frac{m_{k}-1}{m_{k}}\right) \frac{\xi\left(C_{m(k)-1}\right)}{\lambda\left(C_{m(k)-1}\right)}=\frac{\xi\left(C_{m(k)-1}\right)}{\lambda\left(C_{m(k)}\right)} \leq \frac{\xi\left(A_{k}\right)}{\lambda\left(A_{k}\right)} & \leq \frac{\xi\left(C_{m(k)}\right)}{\lambda\left(C_{m(k)-1}\right)} \\
& =\left(\frac{m_{k}}{m_{k}-1}\right) \frac{\xi\left(C_{m(k)}\right)}{\lambda\left(C_{m(k)}\right)} .
\end{aligned}
$$

Thus if $\xi\left(C_{n}\right) / \lambda\left(C_{n}\right) \rightarrow X$ almost surely, then $\xi\left(A_{n}\right) / \lambda\left(A_{n}\right) \rightarrow X$ almost surely as $n \rightarrow \infty$. This proves (ii). From the same estimate we obtain, for large $k$ :

$$
\begin{aligned}
\mathbf{E}\left|\frac{\xi\left(A_{k}\right)}{\lambda\left(A_{k}\right)}-X\right| \leq & \mathbf{E}\left|\frac{\xi\left(A_{k}\right)}{\lambda\left(A_{k}\right)}-\left(\frac{m_{k}}{m_{k}-1}\right) \frac{\xi\left(C_{m(k)}\right)}{\lambda\left(C_{m(k)}\right)}\right| \\
& +\mathbf{E}\left|\left(\frac{m_{k}}{m_{k}-1}\right) \frac{\xi\left(C_{m(k)}\right)}{\lambda\left(C_{m(k)}\right)}-X\right| \\
\leq & \mathbf{E}\left|\left(\frac{m_{k}}{m_{k}-1}\right) \frac{\xi\left(C_{m(k)}\right)}{\lambda\left(C_{m(k)}\right)}-\left(\frac{m_{k}-1}{m_{k}}\right) \frac{\xi\left(C_{m(k)-1}\right)}{\lambda\left(C_{m(k)-1}\right)}\right| \\
& +\mathbf{E}\left|\left(\frac{m_{k}}{m_{k}-1}\right) \frac{\xi\left(C_{m(k)}\right)}{\lambda\left(C_{m(k)}\right)}-X\right| \\
\leq & 2 \mathbf{E}\left|\left(\frac{m_{k}}{m_{k}-1}\right) \frac{\xi\left(C_{m(k)}\right)}{\lambda\left(C_{m(k)}\right)}-X\right| \\
& +\mathbf{E}\left|X-\left(\frac{m_{k}-1}{m_{k}}\right) \frac{\xi\left(C_{m(k)-1}\right)}{\lambda\left(C_{m(k)-1}\right)}\right| .
\end{aligned}
$$

The right-hand side converges to zero as $k \rightarrow \infty$ if $\xi\left(C_{n}\right) / \lambda\left(C_{n}\right) \rightarrow X$ in $L^{1}$ as $n \rightarrow \infty$. This proves (iii).

Lemma 3.13. Let $\xi$ be a random measure on $\mathbb{R}_{+}$that is swap-invariant under a probability measure $\mathbf{P}$. Further let $\left(A_{n}\right)_{n \geq 1}$ be a $\xi$-integrable standard sequence with constant increments and limit $A$ such that $\xi\left(A_{n}\right) / \lambda\left(A_{n}\right) \rightarrow X \mathbf{P}$-almost surely and in $L^{1}(\mathbf{P})$ as $n \rightarrow \infty$ for some random variable $X$ with $\mathbf{E}_{\mathbf{P}} X>0$. Define the random measure $\eta$ by

$$
\eta= \begin{cases}\xi / X & \text { on }\{X>0\} \\ 0 & \text { on }\{X=0\}\end{cases}
$$

and $\mathbf{Q}$ by

$$
\frac{\mathrm{d} \mathbf{Q}}{\mathrm{d} \mathbf{P}}=\frac{X}{\mathbf{E}_{\mathbf{P}} X}
$$

(i) The sequence $\left(\eta\left(\Delta A_{n}\right)\right)_{n>1}$ is exchangeable under $\mathbf{Q}$.

(ii) $\xi\left(A_{n}\right)=0 \mathbf{P}$-almost surely on $\{X=0\}$ for $n \geq 1$. 
(iii) For each $m \geq 1$ and disjoint measurable sets $\left(B_{j}\right)_{1 \leq j \leq m}$ with $\lambda\left(B_{j}\right)=$ $\lambda\left(A_{1}\right), B_{j} \cap A=\emptyset$, and $\mathbf{E}_{\mathbf{P}} \xi\left(B_{j}\right)<\infty$, we have, under $\mathbf{Q}$,

$$
\left(\eta\left(B_{1}\right), \ldots, \eta\left(B_{m}\right)\right) \stackrel{d}{=}\left(\eta\left(\Delta A_{1}\right), \ldots, \eta\left(\Delta A_{m}\right)\right) .
$$

Proof: Apply Corollary 2.18 to the sequence $\left(\xi\left(\Delta A_{n}\right)\right)_{n \geq 1}$. This proves (i) and (ii).

Now choose a sequence $\zeta$ of random variables in $\mathbb{R}_{+}$such that $\mathbf{P}$-almost surely $\zeta_{j}=\xi\left(B_{j}\right)$ for $1 \leq j \leq m$ and $\zeta_{m+j}=\xi\left(\Delta A_{j}\right)$ for $j \geq 1$. Clearly $\zeta$ is swap-invariant under $\mathbf{P}$. We have $n^{-1} \sum_{j=1}^{n} \zeta_{j} \rightarrow \lambda\left(A_{1}\right) X \mathbf{P}$-almost surely and in $L^{1}(\mathbf{P})$. Another application of Corollary 2.18 shows (iii).

Theorem 3.14. Let $\xi$ be a random measure on $\mathbb{R}_{+}$that is swap-invariant under a probability measure $\mathbf{P}$.

(i) For each $\xi$-integrable standard sequence $\left(A_{n}\right)_{n \geq 1}$ there exists an integrable random variable $X$ such that $\xi\left(A_{n}\right) / \lambda\left(A_{n}\right) \rightarrow \bar{X}$ almost surely as $n \rightarrow \infty$.

(ii) Assume that $\mathbf{E}_{\mathbf{P}} \xi$ is $\sigma$-finite and that there exists a standard sequence $\left(A_{n}\right)_{n \geq 1}$ with constant increments and limit $A$ such that $\lambda\left(\mathbb{R}_{+} \backslash A\right)=\infty$, $\xi\left(A_{n}\right) / \lambda\left(A_{n}\right) \rightarrow X$ in $L^{1}(\mathbf{P})$, and $\mathbf{E}_{\mathbf{P}} X>0$. Then $\xi\left(B_{n}\right) / \lambda\left(B_{n}\right) \rightarrow X$ in $L^{1}(\mathbf{P})$ for each standard sequence $\left(B_{n}\right)_{n \geq 1}$.

(iii) Under the same conditions as in (ii), there exists a random measure $\eta$ that is exchangeable under the probability measure $\mathbf{Q}$ defined by

$$
\frac{\mathrm{d} \mathbf{Q}}{\mathrm{d} \mathbf{P}}=\frac{X}{\mathbf{E}_{\mathbf{P}} X}
$$

such that $\xi=X \eta \mathbf{P}$-almost surely.

Notice that under the conditions of (ii) and (iii) each standard sequence is $\xi$ integrable, cf. Theorem 3.6.

Proof of Theorem 3.14: Note that (i) follows by Proposition 3.9 and Lemma 3.12 (i) and (ii).

In order to show (ii), assume that $\left(A_{n}\right), A$, and $X$ have the stated properties, and let $c=\lambda\left(A_{1}\right)$. Now let $\left(B_{n}\right)$ be another standard sequence, say with limit $B$. First we assume that $\left(B_{n}\right)$ has constant increments with $\lambda\left(B_{1}\right)=c$, and that $A \cap B=\emptyset$. Clearly $\mathbf{E}_{\mathbf{P}} \xi\left(B_{n}\right)<\infty$ for all $n$. By swap-invariance

$$
\begin{aligned}
& \mathbf{E}_{\mathbf{P}}\left|n^{-1} \sum_{j=1}^{n} \xi\left(\Delta B_{j}\right)-m^{-1} \sum_{k=1}^{m} \xi\left(\Delta A_{k}\right)\right| \\
& =\mathbf{E}_{\mathbf{P}}\left|n^{-1} \sum_{j=1}^{n} \xi\left(\Delta A_{j}\right)-m^{-1}\left(\sum_{k=1}^{n} \xi\left(\Delta B_{k}\right)+\sum_{k=n+1}^{m} \xi\left(\Delta A_{k}\right)\right)\right|
\end{aligned}
$$

for $m>n \geq 1$. Letting $m \rightarrow \infty$, it follows that

$$
\mathbf{E}_{\mathbf{P}}\left|n^{-1} \sum_{j=1}^{n} \xi\left(\Delta B_{j}\right)-c X\right|=\mathbf{E}_{\mathbf{P}}\left|n^{-1} \sum_{j=1}^{n} \xi\left(\Delta A_{j}\right)-c X\right| .
$$

Letting $n \rightarrow \infty$ shows that $\xi\left(B_{n}\right) / \lambda\left(B_{n}\right) \rightarrow X$ in $L^{1}(\mathbf{P})$, and (i) implies that this convergence is also $\mathbf{P}$-almost surely. Now let $\left(B_{n}\right)$ be an arbitrary standard sequence with limit $B$, i.e. we may have $A \cap B \neq \emptyset$. We may choose a standard sequence with constant increments $\left(E_{n}\right)$ with limit $E$ such that $\lambda\left(E_{1}\right)=c$ and $A \cap E=\emptyset$. By the first part of the proof it follows that $\xi\left(E_{n}\right) / \lambda\left(E_{n}\right) \rightarrow X$ $\mathbf{P}$-almost surely and in $L^{1}(\mathbf{P})$. Now we distinguish the cases $\lambda(A \cap B)<\infty$ and 
$\lambda(A \cap B)=\infty$. In the first case we choose another standard sequence with constant increments $\left(F_{n}\right)$ with limit $F$ such that $\lambda\left(F_{1}\right)=c$ and $F \subset A \backslash B$. Let $\left(C_{n}\right)$ be a CSCI of $\left(B_{n}\right)$ with $\lambda\left(C_{1}\right)=c$, which exists by Lemma 3.12 (i). From the convergence of $\xi\left(E_{n}\right) / \lambda\left(E_{n}\right)$ we consecutively conclude that the same convergence holds for $\left(F_{n}\right)$ and $\left(C_{n}\right)$. Finally $\xi\left(B_{n}\right) / \lambda\left(B_{n}\right) \rightarrow X$ P-almost surely and in $L^{1}(\mathbf{P})$ by Lemma 3.12 (ii) and (iii). In the second case, $\lambda(A \cap B)=\infty$, we may choose a CSCI $\left(C_{n}\right)$ of $\left(B_{n}\right)$ with $\lambda\left(C_{1}\right)=2 c$, and two standard sequences with constant increments $\left(C_{n}^{i}\right)(i=1,2)$ such that, for $n \geq 1$,

$$
C_{n}=C_{n}^{1} \cup C_{n}^{2}, \quad C_{n}^{1} \cap C_{n}^{2}=\emptyset, \quad \lambda\left(\Delta C_{n}^{1}\right)=\lambda\left(\Delta C_{n}^{2}\right)=c .
$$

For $i \in\{1,2\}$ let $C^{i}$ be the limit set of $\left(C_{n}^{i}\right)$. Without loss of generality we may assume that $\lambda\left(A \cap C^{2}\right)=\infty$. Then there is a standard sequence with constant increments $\left(F_{n}\right)$ and limit $F$ such that $\lambda\left(F_{1}\right)=c$ and $F \subset A \cap C^{2}$. By the first part of the proof we consecutively conclude that $\xi\left(F_{n}\right) / \lambda\left(F_{n}\right), \xi\left(C_{n}^{1}\right) / \lambda\left(C_{n}^{1}\right)$, and $\xi\left(C_{n}^{2}\right) / \lambda\left(C_{n}^{2}\right)$ converge to $X \mathbf{P}$-almost surely and in $L^{1}(\mathbf{P})$. It follows that

$$
\frac{\xi\left(C_{n}\right)}{\lambda\left(C_{n}\right)}=\frac{1}{2}\left(\frac{\xi\left(C_{n}^{1}\right)}{\lambda\left(C_{n}^{1}\right)}+\frac{\xi\left(C_{n}^{2}\right)}{\lambda\left(C_{n}^{2}\right)}\right) \rightarrow X \quad \mathbf{P} \text {-a.s. and in } L^{1}(\mathbf{P}) .
$$

Finally we find that $\xi\left(B_{n}\right) / \lambda\left(B_{n}\right) \rightarrow X \mathbf{P}$-almost surely and in $L^{1}(\mathbf{P})$ by Lemma 3.12 (ii) and (iii). This shows statement (ii).

In order to prove (iii) first note that for any $D \in \mathcal{B}\left(\mathbb{R}_{+}\right)$with $\lambda(D)<\infty$ we may choose a standard sequence with constant increments $\left(E_{n}\right)$ such that $E_{1}=D$. Applying Lemma 3.13 (ii) to $\left(E_{n}\right)$ gives $\xi(D)=0 \mathbf{P}$-almost surely on $\{X=0\}$. Now define $\eta$ as in (3.1). It remains to show that $\eta$ is exchangeable under $\mathbf{Q}$. Let $d \in(0, \infty), m \geq 1$, and $\left(B_{j}\right)_{1 \leq j \leq m}$ disjoint measurable sets with $\lambda\left(B_{j}\right)=d$. Define $B=\bigcup_{j=1}^{m} B_{j}$. We may choose a standard sequence $\left(C_{n}\right)$ with constant increments and limit in $\mathbb{R}_{+} \backslash B$ such that $\lambda\left(C_{1}\right)=d$. Statement (ii) implies that $\xi\left(C_{n}\right) / \lambda\left(C_{n}\right) \rightarrow X \mathbf{P}$-almost surely and in $L^{1}(\mathbf{P})$. Applying Lemma 3.13 to the sequence $\left(C_{n}\right)$ and sets $\left(B_{j}\right)_{1 \leq j \leq m}$, we obtain that, under $\mathbf{Q}$,

$$
\left(\eta\left(B_{1}\right), \ldots, \eta\left(B_{m}\right)\right) \stackrel{d}{=}\left(\eta\left(\Delta C_{1}\right), \ldots, \eta\left(\Delta C_{m}\right)\right) .
$$

Now let $\pi \in \operatorname{Perm}(m)$. Deriving the same relation for the permuted sets, we get, under $\mathbf{Q}$,

$$
\left(\eta\left(B_{1}\right), \ldots, \eta\left(B_{m}\right)\right) \stackrel{d}{=}\left(\eta\left(B_{\pi(1)}\right), \ldots, \eta\left(B_{\pi(m)}\right)\right) .
$$

By Kallenberg (1983), Lemma 9.0, this shows that $\eta$ is exchangeable under $\mathbf{Q}$.

Example 3.15. In Example 3.4 let $S=\mathbb{R}_{+}$. Since $\xi$ satisfies the conditions of Theorem 3.14 (ii) (see Examples 3.7 and 3.10), it follows that $\xi\left(B_{n}\right) / \lambda\left(B_{n}\right) \rightarrow X$ $\mathbf{P}$-almost surely and in $L^{1}(\mathbf{P})$ for each standard sequence $\left(B_{n}\right)_{n \geq 1}$.

Note that if $X$ in Theorem 3.14 (ii) is almost surely constant, then $\xi$ is exchangeable under $\mathbf{P}$, similarly to Molchanov et al. (2014), Corollary 24, for random sequences. For easier comparison with existing results we state a variant of Theorem 3.14 for exchangeable random measures.

Theorem 3.16. Let $\xi$ be an exchangeable random measure on $\mathbb{R}_{+}$.

(i) For each $\xi$-integrable standard sequence $\left(A_{n}\right)_{n \geq 1}$ there exists a random variable $X$ such that $\xi\left(A_{n}\right) / \lambda\left(A_{n}\right) \rightarrow X$ almost surely and in $L^{1}$ as $n \rightarrow \infty$.

(ii) Assume that there is $C \in \mathcal{B}\left(\mathbb{R}_{+}\right)$with $\lambda(C) \in(0, \infty)$ and $\mathbf{E} \xi(C)<\infty$. Then the limit in (i) is unique for all standard sequences. 
Proof: Statement (i) is a consequence of de Finetti's theorem, see e.g. Kallenberg (2005), Theorem 1.1. Under the conditions of (ii) $\mathbf{E} \xi$ is $\sigma$-finite and each standard sequence is $\xi$-integrable. Clearly there exists a standard sequence $\left(A_{n}\right)_{n \geq 1}$ with unit increments and limit $A$ such that $\lambda\left(\mathbb{R}_{+} \backslash A\right)=\infty$. By (i) there exists a random variable $X$ such that $\xi\left(A_{n}\right) / \lambda\left(A_{n}\right) \rightarrow X$ almost surely and in $L^{1}$. Now let $\left(B_{n}\right)_{n \geq 1}$ be another standard sequence, say with limit $B$. We may assume that $\left(B_{n}\right)$ has unit increments and that $A \cap B=\emptyset$. The general case is then proven as in Theorem 3.14. Define random sequences $\xi_{j}=\xi\left(\Delta A_{j}\right)$ and $\xi_{j}^{*}=\xi\left(\Delta B_{j}\right)$ for $j \geq 1$, and $g(x)=\lim \sup _{n \rightarrow \infty} n^{-1} \sum_{j=1}^{n} x_{j}$ for $x \in \overline{\mathbb{R}}_{+}^{\infty}$. By the exchangeability of $\xi$

$$
\left(\xi_{1}, \ldots, \xi_{m}, \xi_{m+1}, \ldots\right) \stackrel{d}{=}\left(\xi_{1}^{*}, \ldots, \xi_{m}^{*}, \xi_{m+1}, \xi_{m+2}, \ldots\right),
$$

for $m \geq 1$, and consequently

$$
\begin{aligned}
\left(\xi_{1}, \ldots, \xi_{m}, g(\xi)\right) & \stackrel{d}{=}\left(\xi_{1}^{*}, \ldots, \xi_{m}^{*}, g\left(\xi_{1}^{*}, \ldots, \xi_{m}^{*}, \xi_{m+1}, \xi_{m+2}, \ldots\right)\right) \\
& =\left(\xi_{1}^{*}, \ldots, \xi_{m}^{*}, g(\xi)\right) \quad \text { a.s. }
\end{aligned}
$$

It follows that $(\xi, g(\xi)) \stackrel{d}{=}\left(\xi^{*}, g(\xi)\right)$. Now Kallenberg (2002), Corollary 6.11, implies that $g(\xi)=g\left(\xi^{*}\right)$ almost surely.

By Theorem 3.16 any exchangeable random measure $\xi$ on $\mathbb{R}_{+}$with $\mathbf{E} \xi([0,1])<\infty$ satisfies the ergodic theorem with unique limit. A similar result follows from Nguyen and Zessin (1979). For $\xi$ is clearly stationary, cf. Kallenberg (2002), p. 189. Hence the ergodic theorem Kallenberg (2002), Corollary 10.19, implies that $\xi\left(A_{n}\right) / \lambda\left(A_{n}\right)$ $\rightarrow X$ almost surely and in $L^{1}$ as $n \rightarrow \infty$ for a certain subclass of standard sequences, namely all sequences of increasing bounded convex Borel sets $\left(A_{n}\right)_{n \geq 1}$ such that the inner radius $r\left(A_{n}\right) \rightarrow \infty$.

\section{Acknowledgements}

The author is very grateful to Ilya Molchanov for guidance through the topic and numerous fruitful discussions, and to the referee for several useful comments.

\section{References}

Clyde D. Hardin, Jr. Isometries on subspaces of $L^{p}$. Indiana Univ. Math. J. 30 (3), 449-465 (1981). MR611233.

O. Kallenberg. Random measures. Akademie-Verlag, Berlin; Academic Press, Inc. [Harcourt Brace Jovanovich, Publishers], London, third edition (1983). ISBN 0-12-394960-2. MR818219.

O. Kallenberg. Foundations of modern probability. Probability and its Applications (New York). Springer-Verlag, New York, second edition (2002). ISBN 0-38795313-2. MR1876169.

O. Kallenberg. Probabilistic symmetries and invariance principles. Probability and its Applications (New York). Springer, New York (2005). ISBN 978-0387-25115-8; 0-387-25115-4. MR2161313.

I. Molchanov, M. Schmutz and K. Stucki. Invariance properties of random vectors and stochastic processes based on the zonoid concept. Bernoulli 20 (3), 12101233 (2014). MR3217442.

F. Nagel. Swap-invariant and exchangeable random measures. ArXiv Mathematics e-prints (2016). arXiv: 1602.07666. 
X.-X. Nguyen and H. Zessin. Ergodic theorems for spatial processes. Z. Wahrsch. Verw. Gebiete 48 (2), 133-158 (1979). MR534841. 